\title{
EFICIENCIA Y SELECTIVIDAD DE CARNADA Y ANZUELO EN EL USO DEL PALANGRE PARA LA CAPTURA DEL PEZ “CABEZA DE TORNILLO" ESPINA LARGA (Sebastolobus altivelis) EN AGUAS PROFUNDAS DE BAJA CALIFORNIA, MÉXICO
}

\author{
EFFICIENCY AND SELECTIVITY OF BAIT AND HOOKS IN THE USE OF THE LONGLINE \\ THE CATCH OF TORNYHEAD LONG SPINE (Sebastolobus altivelis) IN DEEP WATERS \\ OF BAJA CALIFORNIA, MÉXICO
}

Jorge Flores Olivares 무, Alfredo Emmanuel Vázquez Olivares @ y Antonio Murillo Olmeda

\begin{abstract}
RESUMEN
En este estudio se determinó la eficiencia y selectividad del palangre de fondo con dos tipos de anzuelos y de carnadas para la captura del pez de profundidad Sebastolobus altivelis, el cual puede representar una pesquería de gran potencial en la zona occidental de Baja California, por su valor y aceptación en el mercado gourmet norteamericano y asiático. El trabajo se realizó de julio de 2012 a marzo de 2013, en la costa occidental de Baja California, entre los 457 y los $1100 \mathrm{~m}$ de profundidad, empleando un palangre provisto de anzuelos de tipo circular plano $10 / 0$ y $11 / 0$ y sardina y anchoveta como carnada. Para determinar la eficiencia de los anzuelos y tipo de carnada se usó la relación de las capturas nominales por cada cien anzuelos, captura por unidad de esfuerzo (CPUE), y la selectividad a partir de una curva de capturas linearizada. A 608 organismos se les midió la longitud y el peso. El rango de tallas fue de 18 a $32 \mathrm{~cm}$ y el peso entre 110 y $400 \mathrm{~g}$. No se encontró diferencia significativa $(\mathrm{P}>0,05)$ entre la eficiencia de los dos tipos de anzuelos con una CPUE de 0,958 y 0,716 (kg/100 anzuelos) para el 11/0 y 10/0, respectivamente, ni entre el tipo de carnada con una CPUE 0,969 y 1,131 para sardina y anchoveta correspondientemente. El análisis de selectividad demostró que la talla de retención L50\% para el anzuelo 11/0 fue de 291,9 mm y para el 10/0 de 302,1 $\mathrm{mm}$. Se determinó que para la efectividad de la pesca de esta especie con estas dimensiones comerciales es indistinto utilizar palangres de fondo con anzuelos 10 y 11 y/o sardina y anchoveta como carnada. Sin embargo, se recomienda el uso de anzuelos de mayor tamaño como medida para su preservación por la alta longevidad.
\end{abstract}

PALABRAS CLAVE: pesca experimental; aguas profundas; selectividad; palangre; anzuelo; Sebastolobus altivelis

\begin{abstract}
This study was determined the efficiency and selectivity of the bottom-set longlines with two types of hooks and types of baits for deep-sea fishing Sebastolobus altivelis, which can represent a large fishery potential in the western area of Baja California, due to its value and acceptance in the North American and Asian gourmet market. The work was carried out from July 2012 to March 2013, on the western coast of Baja California, at depths between 457 to $1100 \mathrm{~m}$, using a longline provided with flat-type hooks 10/0 and 11/0 and sardine and anchovy as bait. To determine the efficiency of hooks and bait type, the ratio of nominal catches was used for each hundred hooks, catch per unit of effort (CPUE), and selectivity from a linearized catch curve. To 608 organisms was measured for length and weight. The size range was 18 to $32 \mathrm{~cm}$ and the weight was between $110 \mathrm{and} 400 \mathrm{~g}$. We did not find a significant difference $(\mathrm{P}>0.05)$ between the efficiency of the two types of hooks with a CPUE of 0.958 and 0.716 (kg/100 hooks) for 11/0 and 10/0 respectively, nor between the type of bait with a CPUE 0.969 and 1.131 for sardine and anchovy correspondingly. The analysis of selectivity showed that the size of retention L50\% for the hook $11 / 0$ was $291.9 \mathrm{~mm}$ and 10/0 of $302.1 \mathrm{~mm}$. It was determined that, for the effectiveness of the fishing of these species, with these commercial dimensions, is indistinct to use bottom-set longlines with hooks 10 and 11 and/or sardine and anchovy as bait. However, the use of larger hooks is recommended as a measure for its preservation due to its high longevity.
\end{abstract}

KEY WORDS: experimental fisheries; deep fisheries; selectivity; longline hook; bait; Sebastolobus altivelis

Dirección de los autores:

Departamento de Posgrado e Investigación, Tecnológico Nacional de México, Instituto Tecnológico de Mazatlán, Calle Corsario I No. 203, Colonia Urías. C.P. 82070. Mazatlán, Sinaloa, e-mail: jfloresolivares1954@gmail.com (J.F.O.); e-mail: alfredoemma@yahoo.com.mx (A.E.V.O); e-mail: murilloolmeda@yahoo.com.mx (A.M.O). 


\section{INTRODUCCIÓN}

Dada la poca evolución en los últimos años de las pesquerías mexicanas, tanto en volumen como en valor (INP, 2004; Csirke, 2005; Csirke et al., 2005) y bajo la óptica de acceder sustentablemente a pesquerías emergentes, el Instituto Tecnológico de Mazatlán, México desarrolló desde el 2006 un programa para la pesca exploratoria y experimental, con la finalidad de orientar la captura hacia especies de aguas profundas de la costa occidental de Baja California, y bajo la modalidad de Pesca de Fomento (Flores et al., 2009) como lo sería el pez cabeza de tornillo espina larga (Sebastolobus altivelis).

Esta especie se caracteriza por tener un gran mercado internacional y aceptación con fines religiosos entre los japoneses, ya que se utiliza en las bodas como símbolo de buena suerte, y también por el sabor de su carne se ha extendido en toda la comunidad oriental que vive en los Estados Unidos, alcanzando precios por producto vivo de hasta 20 dólares la libra. El pez cabeza de tornillo es una especie endémica de las aguas profundas del límite de Estados Unidos con México y puede ofrecer una alternativa pesquera viable para los pescadores que paulatinamente están siendo desplazados por las pesquerías tradicionales de la región de Playas de Tijuana, Pescadero, Cristo Rey, y La Misión en el estado de Baja California. Sin embargo, por las condiciones del recurso en zonas de gran profundidad, aún se desconocen parámetros técnicos que permitan realizar de manera efectiva y selectiva su captura. Por ello, es importante realizar trabajos con pesca experimental con el fin de optimizar las artes de captura que permitan asegurar la sustentabilidad del recurso pesquero.

Taxonómicamente la especie Sebastolobus altivelis pertenece a la familia Scorpanenidae, caracterizada morfológicamente por tener espinas dorsales, anales y pélvicas venenosas, además de su fertilización interna de los huevos (Love et al., 2002). El género Sebastolobus, presente en la costa del Pacífico norte, incluye dos especies más; el pez cabeza de tornillo espina corta $S$. alascanus muy abundante desde el norte de las islas Kuril hasta el sur de California y el pez cabeza de tornillo aleta ancha S. macrochir (Love et al., 2002). El pez cabeza de tornillo espina larga (Sebastolobus altivelis) morfológicamente, cuenta con ojos grandes; color rojizo con tonalidades negras en las aletas; cuerpo alargado; la aleta dorsal exhibe de 15 a 16 espinas, 8-9 de las cuales son más cortas, la tercera es la más larga y con espinas agudas en la región de la cabeza (Love et al., 2002). Se distribuye en el Pacífico norte de la costa de América desde las islas Aleutianas y las Shumagin en el golfo de Alaska hasta Cabo San Lucas, en Baja California Sur, México. En la costa de California se ha registrado en profundidades de los 201 a $1756 \mathrm{~m}$ pero típicamente se encuentra de los 500 a los $1300 \mathrm{~m}$ (Love et al., 2002). Hasta el 83 \% de la población se encuentra en esta zona, de acuerdo a Wakefield (1990), aunque Jacobson y Vetter, (1996), lo han registrado en las costa de Canadá hasta los $1400 \mathrm{~m}$.

Esta especie está adaptada a las condiciones de aguas profundas, caracterizadas por valores mínimos de oxígeno disuelto (Siebenaller, 1978; Siebenaller y Somero, 1982), adoptando una fase adulta sedentaria con desarrollo plantónico larval y pelágico de los juveniles (Smith y Brown, 1983) y con un metabolismo extremadamente bajo resistiendo sin comer por un periodo de hasta 180 días (Vetter y Lynn, 1997). Los juveniles se alimentan probablemente de eufásidos y los adultos de estrellas de mar, cangrejos y mictófidos (Smith y Brown ,1983) con episodios de canibalismo sobre los organismos pequeños (Jacobson y Vetter, 1996). La distribución de las tallas con respecto a la profundidad es relativamente uniforme (Rogers et al., 1997) aunque durante su ciclo de vida los juveniles pueden ser encontrados en aguas someras (Smith y Brown, 1983). Wakefield y Smith (1990) estimaron la talla de primera madurez en 150 mm aproximadamente a los 18 años de edad y no existe un dimorfismo sexual (Starr y Haigh, 2000). El desove ocurre generalmente desde febrero a mayo con un pico en abril (Pearcy, 1962; Best, 1964; Moser, 1974; Wakefield y Smith, 1990) y de acuerdo a Wakefield (1990) y Jacobson y Vetter (1996) de enero a abril en la costa occidental de Estados Unidos. Pearson y Gunderson (2003) reportaron un máximo de desove a los $1000 \mathrm{~m}$ de profundidad. Las larvas se alejan de la superficie pero permanecen en los primeros $200 \mathrm{~m}$ durante $6-7$ meses. Los juveniles permanecen en la zona meso pelágica $(\sim 600 \mathrm{~m})$ por un año con una longitud de hasta $69 \mathrm{~mm}$. Después de la fase pelágica los juveniles alcanzan una longitud total promedio de $55 \mathrm{~mm}$ y tienden a establecerse directamente en el bentos con el resto de la población adulta entre los 600 y 1200 m, aunque según Moser (1974) esto sucede cuando los organismos tienen una longitud de 4,2-6,0 cm y, de acuerdo a Smith y Brown (1983), la máxima abundancia se presenta a los $600 \mathrm{~m}$. Su reclutamiento ocurre cuando alcanzan la talla de $170 \mathrm{~mm}$ (Haigh y Schnute, 2003). Al final de su desarrollo como adultos entre los 24 y 45 años pueden alcanzar una talla de 30 
cm (Ianelli et al., 1994; Jacobson y Vetter, 1996; Love et al., 2002) y de hasta $38 \mathrm{~cm}$ siendo raros los organismos de esta talla (Orr et al., 1998).

El palangre es un arte de pesca tradicional que ha tenido una gran difusión en muchas regiones, sobre todo porque son eficientes en la captura orientadas a la protección de especies depredadoras y que habitan en fondos rocosos (Jacobsen y Joensen, 2004; Fay, 2006). Por otro lado, existe una tendencia internacional al uso del anzuelo curvo, sin embargo se requiere examinar su efecto en la eficiencia del palangre sobre la especie objeto de captura y consecuentemente en la captura por unidad de esfuerzo (CPUE) (Yokota et al., 2006). Al respecto se han realizado estudios sobre la selectividad y eficiencia con palangre de superficie usando diferentes tipos y tamaños de anzuelos entre los que se encuentran los anzuelos curvos y las características de la carnada (Erzini et al., 1997). Tradicionalmente en México se han utilizado los anzuelos rectos, el tipo atunero japonés plano e inclinado, pero por disposición de la norma NOM-029-PESC-2006 de México, se deben usar anzuelos curvos cuando menos en los reinales más cercanos a cada orinque, (Figura 1), considerando que esta medida reduce la pesca incidental (Diario Oficial de la Federación, 2007). Sin embargo, los pescadores en México, se resisten al uso de este anzuelo curvo por ser un elemento nuevo y extraño, llevándolos a desconfiar del rendimiento de la pesca.

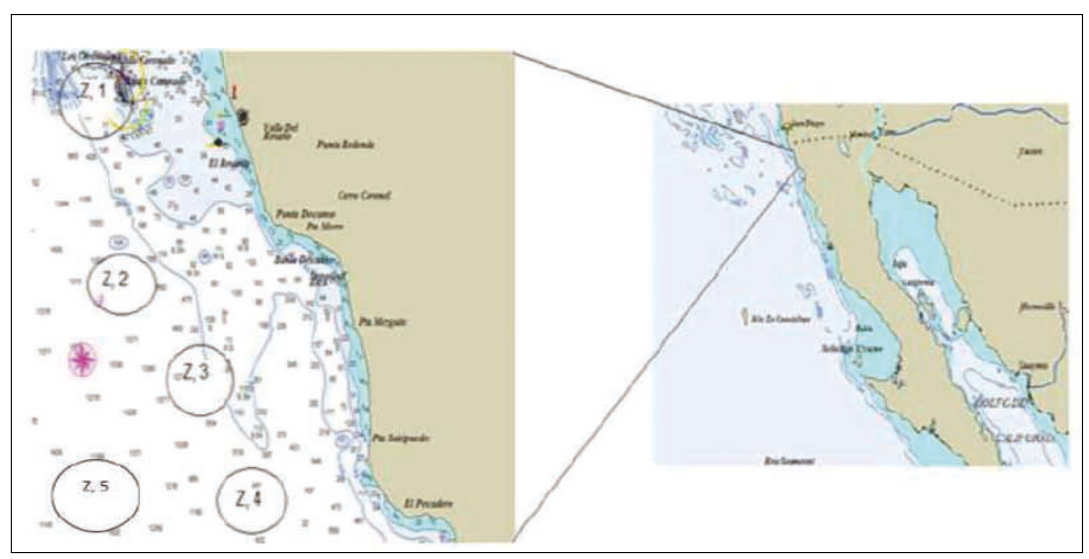

Figura 1. Ubicación geográfica del área de estudio en el océano Pacífico, frente a la costa occidental de Baja California, México.

Con respecto al tipo de carnada empleada en la pesca con palangre pelágico del océano Pacífico, los pescadores han utilizado tradicionalmente calamar (Dosidicus gigas), macarela (Scomber japonicus), lisa (Mugil cephalus) y barrilete (Katsuwonus pelamis), y en la pesca dirigida a peces de roca en México es común utilizar sardina anchoveta, macarela calamar, barrilete y lisa (Santana et al., 1998). Por lo tanto, en el presente trabajo se planteó como objetivo determinar la eficiencia y selectividad de dos tipos de anzuelo y dos tipos de carnada en la pesca del pez cabeza de tornillo espina larga (Sebastolobus altivelis), con palangre de profundidad con la finalidad de contribuir a la generación de información que permita eventualmente su explotación bajo un marco de sustentabilidad, ya que actualmente esta especie no se aprovecha.

\section{MATERIALES Y MÉTODOS}

El área de estudio comprendió desde Coronado $\left(32^{\circ} 23^{\prime} 26^{\prime \prime} \mathrm{N}, 117^{\circ} 23^{\prime} 20^{\prime \prime} \mathrm{O}\right)$ hasta Pescadero $\left(31^{\circ} 55^{\prime} 31^{\prime} \mathrm{N}, 117^{\circ} 04^{\prime} 12^{\prime \prime} \mathrm{O}\right)$, figura 2, Baja California Norte, incluyendo un total de 5 zonas de pesca, debido a que estos sitios son hábitat natural de esta especie y los pescadores ya han iniciado actividades para su captura. Esta zona forma parte del cañón submarino que abarca desde la zona de San Diego, USA, hasta El Bajo de La Misión, Baja California México. Los lances se realizaron en un rango de profundidad de 457-1100 $\mathrm{m}$ coincidiendo con el rango reportado por (Love et al., 2002) de 400-1100 m. Se usó una embarcación menor (eslora: 7,92 m y manga: 1,83 m) provista con un cobra líneas y tanques con aireación para el almacenamiento y 
transporte de producto vivo. El palangre de fondo usado, consistió en una línea madre de polietileno (PE) torcido de $5 \mathrm{~mm}$ de diámetro con una longitud de $1200 \mathrm{~m}$ por sección, con reinales de polietileno (PE) monofilamento de $0,20 \mathrm{~m}$ de largo y $1 \mathrm{~mm}$ de diámetro, separados entre sí por 1,83 m y unidos a la línea madre por medio de un nudo. Contenía 700 anzuelos por sección, donde se unían hasta siete secciones en un mismo punto de largado. Cada extremo de la línea madre contó con un cabo de polietileno torcido de $1200 \mathrm{~m}$ para comunicar la línea en el fondo con la superficie y en cada extremo boyas de señalamiento de $1 \mathrm{~m}$ de diámetro y una banderola de 2 $\mathrm{m}$ de alto. Se probaron anzuelos circulares planos 10/0 y 11/0 de manufactura noruega marca Mustad, con los siguientes datos técnicos: anzuelo 11/0: redondo marca Mustad, (39965D) con recubrimiento Duratin y punta redondeada, ligeramente doblada a la derecha (de 18 a 20 grados); anzuelo 10/0: redondo marca Mustad (39960S) en acero inoxidable con punta redondeada sin ángulo.

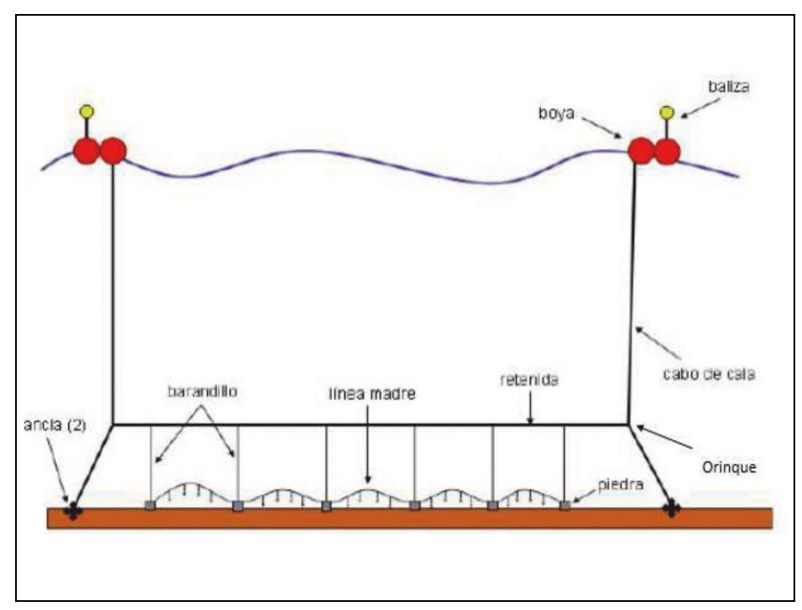

Figura 2. Esquema del palangre experimental.

Cada anzuelo fue encarnado con sardina (Sardinops sagax) o anchoveta (Engraulis mordax Girard), fresca congelada en salmuera para preservar sus propiedades organolépticas.

La captura obtenida en cada línea de anzuelos se contó y pesó en su totalidad. Posteriormente, se determinó la composición por especie de acuerdo a diversas claves (Fischer et al., 1995; Miller y Lea, 1972; INP, 1976; Thomson et al., 1979) y se registró el peso total de la captura y de cada una. A una muestra aleatoria se le determinó la longitud total, utilizando un ictiómetro con una precisión de $\pm 1 \mathrm{~mm}$ y el peso total con una balanza electrónica marca Tosco, modelo 78, con intervalo de medición entre 1 a 2000 g y precisión de \pm 1 g. No se consideró la identificación de sexos ya que en esta especie no se manifiesta un dimorfismo sexual definido (Starr y Haigh, 2000). Los lances experimentales se realizaron durante 3 periodos entre julio del 2012 y marzo del 2013 debido a que estos periodos son los establecidos de acuerdo a los cambios estacionales representativos en el año tales como otoño, invierno y primavera, en cada lance se utilizaron 400 carnadas divididas en cuatro secciones de 100 carnadas de un mismo tipo cada uno. Se combinaron 400 anzuelos y 400 carnadas para formar cuatro combinaciones: anzuelo\#11-sardina, anzuelo\#11-anchoveta, anzuelo\#10 -sardina, anzuelo\#10 -anchoveta en paquetes de 100 anzuelos y 100 carnadas cada combinación, los cuatro paquetes se distribuyeron al azar en cada lance. El proceso para hacerlo aleatorio, necesario para reducir el sesgo causado por la posición de cada combinación en el palangre, consistió en cambiar la posición de cada anzuelo y cada carnada entre las diferentes secciones entre lances de modo que el ciclo se cubrió cada seis lances. El tiempo de reposo de las cimbras se mantuvo en 12 y $24 \mathrm{~h}$ en la mayoría de los lances. Con el total de 608 datos de longitud total colectados durante los muestreos, se elaboró el histograma correspondiente para determinar la distribución de la estructura de la población muestreada por tallas (Zar, 1996). Los datos de peso y longitud total se relacionaron por medio de una regresión de tipo potencial (Bagenal, 1978) ajustada con el método de mínimos cuadrados con un intervalo de confianza del 95 \%, empleando la siguiente ecuación (Sokal y Rohlf, 1981):

$$
P=(a)\left(L t^{b}\right)
$$

Dónde: P es el peso del organismo; Lt la longitud total y b el exponente de la ecuación potencial.

El análisis de la eficiencia de los anzuelos y del tipo de carnada fue determinada con base en los valores de la captura por unidad de esfuerzo (CPUE) expresada mediante la siguiente fórmula:

$$
\text { CPUE = Captura nominal / } 100 \text { anzuelos }
$$

La selectividad fue estimada usando el método de 
selectividad a partir de una curva de capturas linearizada, empleando el programa FISAT ll (FAO/ICLARM Stock Assessment Tools). Se evaluó la normalidad de Kolmogorov -Smirnov y homoscedasticidad de Bartlett de los valores de captura por unidad de esfuerzo (CPUE) obtenidos del empleo de los dos tipos de anzuelos y de los dos tipos de carnada, y para compararlos se aplicó una prueba t-Student (Zar, 1996).

\section{RESULTADOS}

La talla y peso promedio para toda las combinaciones fue de $25,1 \pm 2,0 \mathrm{~cm}$ y $226 \pm 34 \mathrm{~g}$ respectivamente. La talla máxima fue de $32 \mathrm{~cm}$ correspondiendo a un peso de $350 \mathrm{~g}$. La talla máxima fue de $32 \mathrm{~cm}$ correspondiendo a un peso de $350 \mathrm{~g}$. La especie presentó una estructura por tallas unimodal, con un valor modal promedio de 26,3 cm, un mínimo y máximo de 18 y $32 \mathrm{~cm}$, respectivamente (figura 3). En la figura 4 se puede observar la curva ajustada de tipo potencial $(\mathrm{P}<0,05)$ obtenida para la relación talla-peso con un valor de $a=0,8955$ y $b=1,7135$.

En la relación talla-peso el valor de la constante obtenido de $b=1,71$ fue menor que los valores de 3,16 para Columbia Británica (Haigh et al., 2005) y de 3,35 para Oregon (Jacobson, 1991). La eficiencia promedio para ambos anzuelos y carnadas en términos de captura total fue de 1,079 kg, con una CPUE de 1,187 (kg/100 anzuelos), presentado un intervalo un valor mínimo de 0,972 y un máximo de 1,592 (kg/100 anzuelos) (tabla 1).

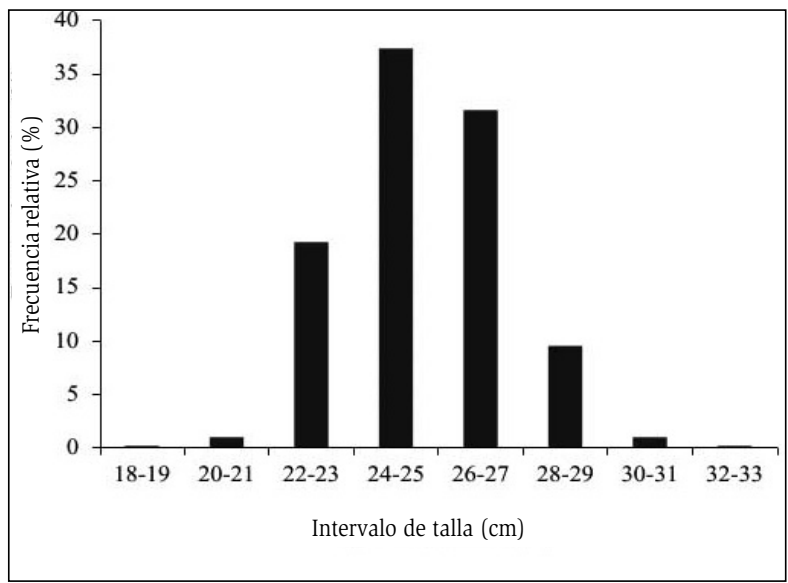

Figura 3. Estructura por tallas del pez cabeza de tornillo espina larga (Sebastolobus altivelis) en la costa occidental de Baja California, para el periodo de julio del 2012 a marzo del 2013.
Tabla 1. Valores de captura total y CPUE obtenidos para Sebastolobus altivelis durante cada periodo de muestreo.

\begin{tabular}{rrr}
\hline Periodo & $\begin{array}{c}\text { Captura total } \\
\text { (kg) }\end{array}$ & $\begin{array}{c}\text { CPUE } \\
\text { (kg/100) } \\
\text { anzuelos) }\end{array}$ \\
\hline 1 & 613 & 1,592 \\
2 & 1265 & 0,972 \\
3 & 1360 & 0,996 \\
Promedio & 1079 & 1,187 \\
\hline D.E. & 407 & 0,351 \\
\hline
\end{tabular}

Como resultado de los experimentos específicos para comparar la eficiencia entre los dos tipos de anzuelos no se encontró una diferencia significativa $(P>0,05)$ con una CPUE de 0,958 y 0,716 (kg/100 anzuelos) para el $11 / 0$ y 10/0, respectivamente. En la figura 5 se puede observar gráficamente los valores de captura total y CPUE obtenidos del experimento para comparar los dos tipos de carnadas sin que tampoco se encontrara una diferencia significativa $(P>0,05)$ entre la eficiencia de la sardina y la anchoveta con una CPUE de 0,969 y 1,131 (kg/100 anzuelos), respectivamente.

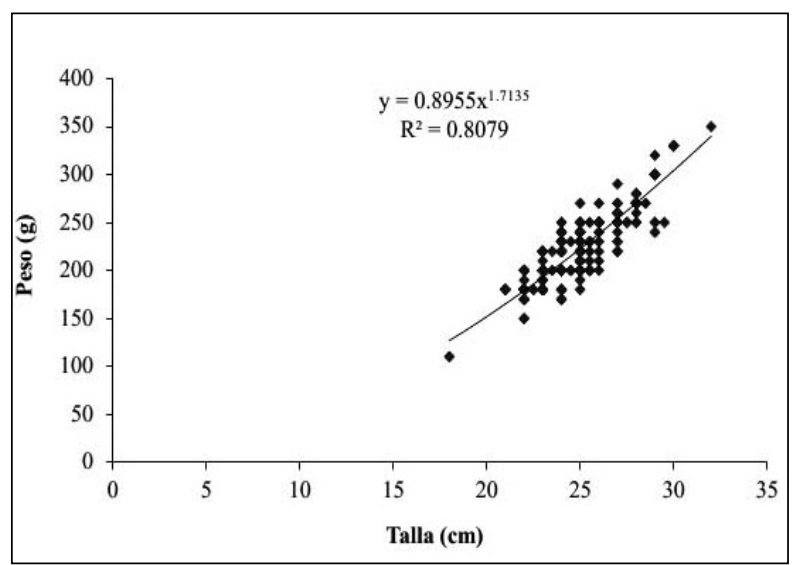

Figura 4. Relación talla-peso del pez cabeza de tornillo espina larga (Sebastolobus altivelis) en la costa occidental de Baja California, para el periodo de julio del 2012 a marzo del 2013.

En la figura 6 se muestran las curvas de selectividad para la captura del pez cabeza de tornillo espina larga (Sebastolobus altivelis) con respecto a la talla en $\mathrm{cm}$ correspondientes a los dos tipos de anzuelos (10/0 y 11/0, Mustad) obteniéndose una talla de retención $\mathrm{L}_{50 \%}$ para el anzuelo $11 / 0$ de $291,9 \mathrm{~mm}$ y para el $10 / 0$ de $302,1 \mathrm{~mm}$. 


\section{DISCUSIÓN}

Los lances se realizaron a un rango de profundidad de 457-1 $100 \mathrm{~m}$ coincidiendo con el rango reportado por (Love et al., 2002) de 400-1100 m (Smith y Brown, 1983; Vetter y Lynn, 1997). El peso promedio de $226 \mathrm{~g}$ para los peces muestreados durante los tres periodos de estudio, fue superior al peso promedio reportado para la pesquería de Canadá de 114 g (Haigh et al., 2005), aunque de acuerdo a los valores de la ecuación potencial de relación talla-peso de estos mismos autores, un organismo de la talla promedio registrada $(25,1 \mathrm{~cm})$ tendría un peso aproximado de $200 \mathrm{~g}$.

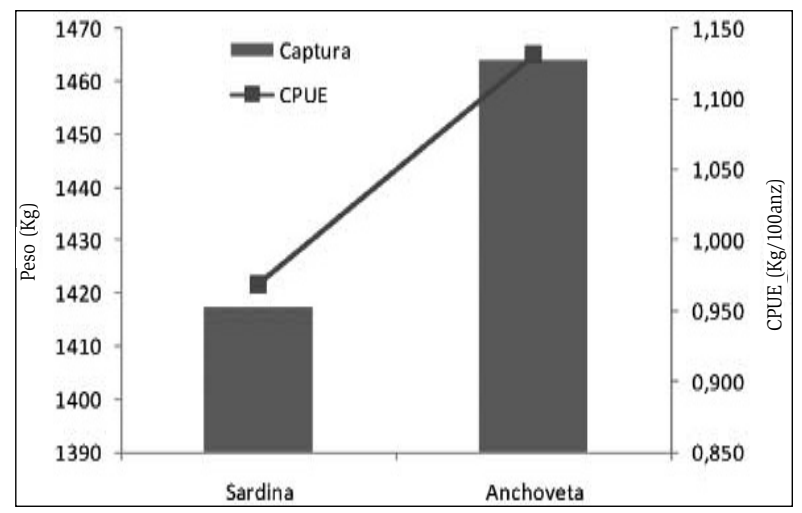

Figura 5. Captura total y CPUE obtenidos en el experimento para comparar la eficiencia entre los dos tipos de carnada (sardina y anchoveta) utilizadas en la captura del pez cabeza de tornillo espina larga (Sebastolobus altivelis).
En el histograma de la estructura por tallas de los peces capturados experimentalmente la moda con un valor de $26,3 \mathrm{~cm}$, figura 3 , que teóricamente correspondería a la última moda de la mayoría de las distribuciones polimodales por tallas para especies de rápido crecimiento, pero en el caso del pez cabeza de tornillo espina larga (Sebastolobus altivelis), esta moda incluye un conjunto de cohortes que agrupan a los organismos adultos más longevos de entre 24 y 45 años que se encuentran al final de su desarrollo (Ianelli et al., 1994; Jacobson y Vetter, 1996; Love et al., 2002). En consecuencia al pescar como se propone actualmente, con base a una talla mínima de captura comercial que estaría incluida en dicha moda, no necesariamente se removería el excedente de la población planteado por el modelo típico de rendimiento sostenible (Beverton y Holt, 1966). Esto se puede corroborar considerando el análisis de las edades obtenidas a partir de las tallas, con la ecuación de crecimiento ( $\mathrm{L} \infty=31,2 \mathrm{~cm}, \mathrm{k}=0,064 \mathrm{y}$ to $=-2,02$ ), registradas para (Sebastolobus altivelis) por Fay (2006) en que según los cálculos, las tallas entre 24 y $32 \mathrm{~cm}$ corresponde a edades entre 21 y 46 años, y longitudes que en la distribución de frecuencia por tallas obtenida para este trabajo (figura 3), representan aproximadamente el $80 \%$ del total de los organismos. Este tipo de distribución es típica para la especie y se ha reportado como relativamente uniforme con respecto a la profundidad (Rogers et al., 1997) posiblemente debido a que después de su asentamiento como adulto no presenta migraciones (Haigh et al., 2005).

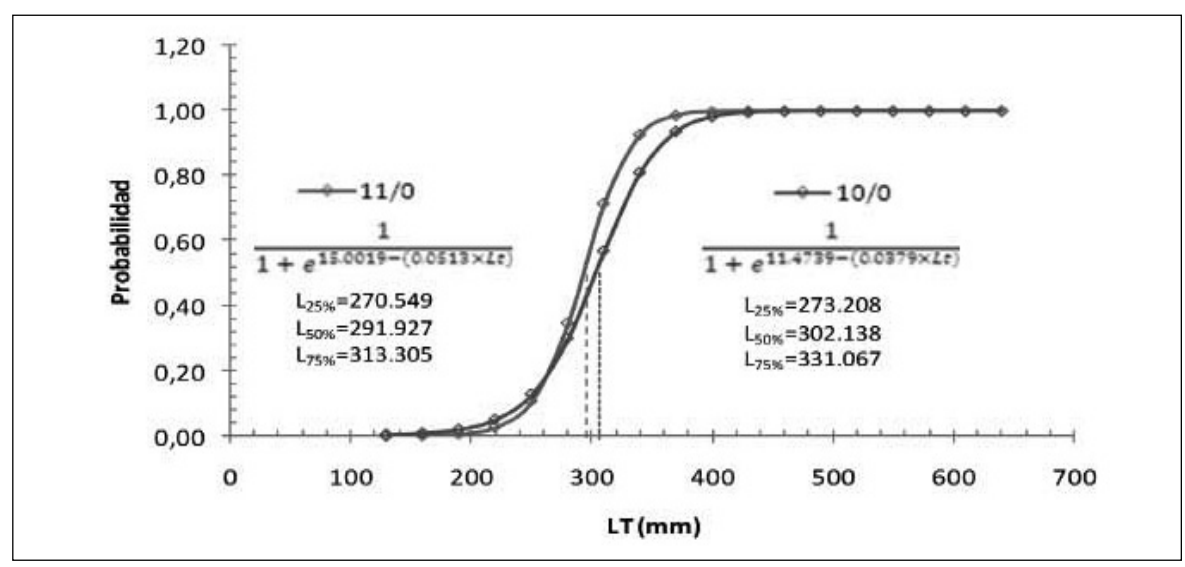

Figura 6. Curvas de probabilidad de captura del pez cabeza de tornillo espina larga (Sebastolobus altivelis) con los anzuelos "Mustad" $10 / 0$ y 11/0. 
Cabe resaltar que la existencia de organismos entre 18 y $19 \mathrm{~cm}$ representa la presencia de peces que apenas estarían alcanzando la talla de primera madurez, ya que se ha estimado que a partir de los 18 años de edad, entre $15 \mathrm{~cm}$ y 18,8 cm, estos organismos alcanzan tal condición (Wakefield y Smith, 1990; Jacobson 1991; Pearson y Gunderson, 2003), por lo que se debería poner atención al tamaño mínimo de anzuelo que se use en los palangres para evitar su captura. Al no presentarse diferencias significativas en la CPUE entre los anzuelos empleados, ni tampoco en la talla de retención de acuerdo al análisis de selectividad, aparentemente el tamaño del anzuelo no influyó en las capturas, concordando con lo ya previamente concluido de que los anzuelos pequeños son casi tan eficientes como los de mayor tamaño para capturar peces grandes (Ralston, 1990; Bjordal y Lokkeborg, 1996). La efectividad y selectividad del palangre depende de la interrelación de factores ambientales, biológicos y tecnológicos (Løkkeborg y Bjordal, 1992) además del índice lunar y la velocidad del viento que pueden afectar la vulnerabilidad del pez a las operaciones de pesca (Bigelow et al., 1999), la profundidad de captura del palangre en relación a la distribución vertical de las especies objeto de captura y las incidentales (Santana et al., 1998), los tiempos de operación del calado, cobrado y deriva del palangre, el tipo de carnada y su posición de encarnado (Broadhusrst y Hazin, 2001), el tipo y tamaño del anzuelo (Kerstetter y Graves, 2006; Yokota et al., 2006).

El intervalo de valores promedio de la CPUE obtenidas para el arte de pesca incluyendo ambos anzuelos y carnadas fue de 0,972 y 1,592 (kg/100 anzuelos) (tabla 2). Al considerar que no existió una diferencia significativa del valor CPUE entre carnadas y anzuelos, ni tampoco con respecto a la talla de retención en términos de selectividad, indicaría que los peces en general, independientemente de su talla, son extremamente vulnerables al arte de pesca empleado (Kimura y Zenger, 1997). El hecho de no presentar preferencia por alguna de las carnadas empleadas puede ser consecuencia de que la carnada en sí representa un alimento atractivo, ya que olor que desprende es una de sus principales características de atracción que permite estimular el comportamiento del pez en busca del alimento y provocar el contacto con el arte de pesca, que en el caso de (Sebastolobus altivelus) es fácilmente accesible y relativamente abundante con respecto a el escaso alimento en su medio ambiente natural (Smith y Brown, 1983; Jacobson y Vetter, 1996; Blaha, 2003).

La atractibilidad de la carnada sin importar el tamaño del anzuelo, hace muy vulnerables a los peces hambrientos que apenas han alcanzado la talla de primera madurez, ya que tienen un diámetro de boca muy similar al de los adultos, lo que les permite engullir anzuelos aparentemente grandes como los empleados en esta investigación. Lo anterior representa una ventaja en cuanto a la disponibilidad de carnadas para el caso de Baja California pues al comparar los costos por tonelada de ambas, la sardina cuesta aproximadamente $20 \%$ más que la anchoveta y por rendimiento en número de carnadas, la anchoveta rinde aproximadamente 2,25 veces más carnadas que la sardina por tonelada.

Esto hace que la anchoveta, en comparación con la sardina represente menor costo y quizá esto sea una de las razones por lo que los pescadores, de manera rutinaria, en la pesca con el palangre de fondo encarnan los anzuelos con anchoveta como carnada principal e intercalan carnadas de sardina cada 4 o 5 anzuelos.

\section{CONCLUSIONES}

No se encontraron diferencias significativas $(\mathrm{P}>0,05)$ entre la eficiencia de los dos tipos de anzuelos con una CPUE de 0,958 y 0,716 (kg/100 anzuelos) para el $11 / 0$ y 10/0, respectivamente. Tampoco se encontraron diferencias significativas $(\mathrm{P}>0,05)$ entre la eficiencia de la sardina y la anchoveta con una CPUE de 0,969 y 1,131 (kg/100 anzuelos), respectivamente. Con base en el análisis de selectividad se determinó que la talla de retención L50\% para el anzuelo 11/0 fue de 291,9 mm y para el $10 / 0$ de $302,1 \mathrm{~mm}$. Se determinó que para la efectividad de la pesca de esta especie con estas dimensiones comerciales es indistinto utilizar palangres de fondo con anzuelos 10 y 11 y/o sardina y anchoveta como carnada. Sin embargo, se recomienda el uso de anzuelos de mayor tamaño como medida para su preservación por su alta longevidad.

\section{REFERENCIAS}

Bagenal, T. 1978. Aspects of fish fecundity. En: Gerking S.D., Editor. Ecology of freshwater fish production. Blackwell Scientific, Oxford, Gran Bretaña.

Best, E. 1964. Spawning of longspine channel rockfish, Sebastolobus altivelis. California fish and game (50):265-267. Beverton, R. y Holt, S. 1966. On the dynamics of exploited fish populations. Fishery Investigations, London, Series 2(19): 533.

Bigelow, K., Boggs, C. y He, X., 1999. Environmental effects on swordfish and blue shark catch rate in the US North Pacific 
longline fishery. Fisheries Oceanography 8 (3): 178-198. Bjordal, y Løkkeborg. S. 1996. Longlining. Fishing News Books, Blackwell Science, London.

Blaha, F. 2003. Bait for longlining fishery on snapper (Pagrus auratus). Tesis de Maestría, University of Auckland, Auckland, New Zealand.

Broadhurst, M. y Hazin, H. 2001. Influences of type and orientation of bait on catch of swordfish (Xiphias gladius) and other species in artisanal sub-surface longline fishery off northeastern Brazil. Fisheries Research (53): 169-179.

Csirke, J. 2005. Producción y situación mundial de los recursos pesqueros marinos. En: FAO. Editor. 2005. Examen de la situación de los recursos pesqueros marinos mundiales. Documento Técnico de Pesca FAO, Roma.

Csirke, J., Gumy, A., Lleonart, J., González de la Rocha, J., Seijo, J.C., Sosa, E. y Martínez-Cordero, F.J. 2005. Evaluación para el fortalecimiento del Instituto Nacional de la Pesca. FAO, Roma.

Diario Oficial de la Federación, 2007. Norma oficial mexicana. NOM-029-PESC-2006. Pesca responsable de tiburones y rayas. Especificación para su aprovechamiento. Secretaría de Gobernación, México D.F.

Erzini, K., Gonçalves, J., Bentes, L., Lino, P. y Ribeiro, J. 1997. Longline selectivity and biology of "red" seabreams (Sparidae). Final Report, Project Ref. Besugo C/1 94/059. European Commission, DGXIV Fisheries, Universida de do Algarve, UCTRA.

Fay, G. 2006. Stock Assessment and Status of Longspine Thornyhead (Sebastolobus altivelis) off California, Oregon and Washington in 2005 Draft for September PFMC Meeting 30th August 2005. School of Aquatic and Fishery Sciences, University of Washington, Washington D.C.

Fischer, W., Krupp, F., Schneider, W., Sommer, C., Carpenter, K. y Niem, V. 1995. Guía FAO para la identificación de especies para los fines de la pesca. Pacifico centro-oriental. Volumen III. Vertebrados - Parte 2. FAO. Roma.

Flores, J., Chávez, O. y Jiménez, Q. 2009. Captura de Eptatretus stoutii frente a la Costa Noroccidental de la Baja California, México, 2006-2008. Ciencia Pesquera 2 (17) 53-64.

Haigh, R. y Schnute, J. 2003. The Longspine Thornyhead fishery along the west coast of Vancouver Island, British
American Journal of Fisheries Management (23): 120-140. Haigh, R., Olsen, N. y Starr, P. 2005. A review of longspine thornyhead Sebastolobus altivelis along the Pacific coast of Canada: Biology, distribution, and abundance trends. Canadian stock assessment advisory. Research document 2005/097, Fisheries and Oceans, Otawwa.

Ianelli, J., Lauth, R. y Jacobson, L., 1994. Status of the thornyhead (Sebastolobus sp.) resource in 1994. Appendix D. En: Status of the Pacific coast groundfish fishery through 1994 and recommended acceptable biological catches for 1995. Editor. Appendix (1): Stock assessment and fishery evaluation. Pacific Fishery Management Council, Portland, Oregon.

INP, 1976. Catálogo de Peces Marinos Mexicanos. Secretaría de Industria y Comercio. Subsecretaria de Pesca, Instituto Nacional de la Pesca. México, D. F., México.

INP, 2004. Carta Nacional Pesquera. Diario Oficial de la Federación. Subsecretaria de Pesca, Instituto Nacional de la Pesca. México, D. F., México.

Jacobson, L. 1991. Thornyheads stock assessment for 1991. En: appendices to the Status of the Pacific coast groundfish fishery through 1990 and recommended acceptable biological catches for 1991. Appendix (1): Stock Assessment and fishery evaluation. PFMC. Portland, Oregon.

Jacobsen, J. y Joensen, J. 2004. Comparison of bait in longline fishery. Tesis Biologia, University of Faroe Island, Faroe Island, Denmark.

Jacobson, L. y Vetter, R. 1996. Bathymetric demography and niche separation of thornyhead rockfish: Sebastolobus alascanus and Sebastablobus altivelis. Canadian Journal of Fisheries and Aquatic Sciences (53): 600-609.

Kerstetter, D. y Graves, J. 2006. Effects of the circle versus J-style hooks on target and no-target species in a pelagic longline fisheries. Fisheries Research (80): 239-250.

Kimura, D. y Zenger, H. 1997. Standardizing sablefish (Anoplopoma fimbria) long-line survey abundance indices by modeling the log-ratio of paired comparative fishing cpues. ICES, Journal of Marine Science (54): 48-59.

Løkkeborg, S. y Bjordal, Å. 1992. Species and size selectivity in long line fishing: a review. Fisheries Research (13): 311-322. Love, M., Yoklavich, M. y Thorsteinson, L. 2002. The Rockfishes of the Northeast Pacific. University of California Press, Berkley, California.

Miller, D. y Lea, R., 1972. Guide to the coastal marine fishes of California. Fish Bullettin 157, Department Fish and Game, California. 
Moser, H. 1974. Development and distribution of larvae and juveniles of Sebastolobus (Pisces; family Scorpaenidae). Fishery Bulletin (72): 865-884.

Orr, J., Brown, M. y Baker, D. 1998. Guide to Rockfishes (Scorpaenidae) of the Genera Sebastes, Sebastolobus, and Adelosebastes of the Northeast Pacific Ocean. NOAA Technical Memorandum NMFS-AFSC-95, Seattle.

Pearcy, W. 1962. Egg masses and early developmental stages of the scorpaneid fish, Sebastolobus. Journal of the Fisheries Research Board of Canada (19): 1169-1173.

Pearson, K. y Gunderson, D., 2003. Reproductive biology and ecology of shortspine thornyhead rockf ish, Sebastolobus alascanus, and longspine thornyhead rockfish, S. altivelis, from the northeastern Pacific Ocean. Environmental Biology of Fishes (67):11 - 136.

Ralston, S., 1990. Size selection of snappers (Lutjanidae) by hook and line gear. Canadian Journal of Fisheries and Aquatic Sciences (47): 696-700.

Rogers, J., Jacobson, L., Lauth, R., Ianelli, J. y Wilkins, M. 1997. Status of the Thornyhead (Sebastolobus sp.) Resource in 1997. En: Pacific Fishery Management Council. 1997. Appendix: Status of the Pacific Coast Groundfishery Through 1997 and Recommended Biological Catches for 1998: Stock Assessment and Fishery Evaluation. Pacific Fishery Management Council. Portland, Oregon.

Santana, H., Macías, R. y Valdez, J. 1998. Selectividad del sistema de palangre utilizado por la flota mexicana en la zona económica exclusiva. Ciencias Marinas (24): 193-210.

Siebenaller, J., 1978. Genetic variability in deep-sea fishes of the genus Sebastolobus (Scorpaenidae). En: Battaglia, B. y Beardmore, J.A. Editor. Marine organisms: genetics, ecology and evolution. Plennum Press, New York.

Siebenaller, J. y Somero, G. 1982. The Maintenance of different enzyme activity levels in congeneric fishes living at different depths. Physiological Zoology (55): 171-179.
Smith Jr., K. y Brown, N. 1983. Oxygen consumption of pelagic juveniles and demersal adults of the deep-sea fish Sebastolobus altivelis, measured at depth. Marine Biology (76): 325-332.

Sokal, R. y Rohlf, F. 1981. USA, Freeman, Biometry: the principles and practice of statistics in Biological Research. Freeman and Company, New York.

Starr, P. y. Haigh, R. 2000. Assessment of the Canadian longspine thornyhead (Sebastolobus altivelis) for 2000. Canadian Stock Assessment Secretariat. Nanaimo, British Columbia.

Thomson, D., Findley, L. y Kerstitch, A., 1979. Reef Fishes of the Sea of Cortez. Wiley, Nueva York,

Vetter, R. y Lynn, E. 1997. Bathymetric demography, enzyme activity patterns, and bioenergetics of deep-living scorpaenid fishes (genera Sebastes and Sebastolobus): paradigms revisited. Marine Ecology Progress Series (155): 173-188.

Wakefield, W. 1990. Patterns in the distribution of demersal fishes on the upper continental slope off central California with studies on the role of ontogenetic vertical migration in particle flux. Tesis de doctorado, University of California, San Diego, CA, Estados Unidos de América.

Wakefield, W. y Smith. Jr, K. 1990. Ontogenetic vertical migration in Sebastolobus altivelis as a mechanism for transport of particulate organic matter at continental slope depths. Limnology and Oceanography (35): 1314-1328.

Yokota, K., Kiyota, M. y Minami, H., 2006. Shark catch in a pelagic longline fishery: comparison of circle and tuna hooks. Fisheries Research (81): 337-341.

Zar, J.1996. Biostatistical analysis. (3a Ed.). Englewood Cliffs, N.J., Prentice-Hall Inc, New Jersey.

Fecha de recepción: 29/11/2017

Fecha de aceptación: 16/04/2018

Publicado en línea: 28/05/2018

Para citar este artículo: Flores Olivares J., Vázquez Olivares A.F. y Murillo Olmeda, A. 2018. Eficiencia y selectividad de carnada y anzuelo en el uso del palangre para la captura del pez "cabeza de tornillo" espina larga (Sebastolobus altivelis) en aguas profundas de Baja California, México. Intropica 13(1): 31-40. DOI: http://dx.doi.org/ 10.21676/23897864.2380 
